\title{
Semantic-based Arabic Question Answering: Core and Recent Techniques
}

\author{
Yousif Haroon Abaker ${ }^{1}$ and Mohsen Rshwan ${ }^{2}$ \\ ${ }^{1}$ Computer Science and Information Technology College, Sudan University of \\ Science and Technology, Khartoum, Sudan \\ ${ }^{2}$ Electronics and Communications Department, Cairo University, Egypt \\ ${ }^{1}$ usif.gm@gmail.com, ${ }^{2}$ mrashwan@rdi-eg.com
}

\begin{abstract}
Providing the right answer remains a hot research field in Question Answering (QA). Considerable improvements in QA performance were obtained by adopting core semantic techniques like WordNet. However, an ideal adoption of such technique in Arabic QA is quite challenging, particularly for Fatwa QA which answers questions in Islamic religion domain. The point of this review is to overview the state of the art Arabic Fatwa QA models with a focus on the core and recent semantic representation techniques and present a comparative summary that involves $Q A$ paradigms, semantic techniques, performance, scope, and limitations. The Aim is to highlight the new directions and open issues regarding the challenges for answering Arabic Fatwa questions on semantics basis. Recent semantic techniques like "Skip-Gram/CBOW" $N N$ embeddings revealed a promising performance compared to a core technique "Ontology" when adopted in some tasks of Arabic Fatwa QA.
\end{abstract}

Keywords: Question Answering; Fatwa; Semantic representation; embeddings; Semantic Composition; Ontology; LSA; CBOW/SKIP-G; Eigenwords

\section{Introduction}

Arabic Question Answering (QA) that manipulates various forms of Arabic language including the formal written and spoken language Modern Standard Arabic (MSA), the language of Quran and Hadith Classical Arabic (CA) and the local speech-language Dialectical Arabic (DA), like "Fatwa" did not take much attention in the research field of Arabic QA [1]. Fatwa is a restricted domain Question Answering (QA) about the legal rulings and opinions in Islam. The aim is to satisfy the continuous Muslims demands to learn, to remind or to study what is legitimate in Islam. In this paper, (FQA) stand for Fatwa Question Answering [2].

In Fatwa domain, there is a large repository of archived answers in form of questionanswer pairs, those answers are prepared by an authorized expert "Mufti" [3-4] or an official Fatwa organization [5-6]. FQA systems utilize this reliable source of information to answer new but similar questions posed in Natural Language (NL). However, there are some challenges that face Arabic FQA systems which include:

- The Answers must be at a high degree of precision in the religious domain [2-7].

- The semantic gap between the new Fatwa question that posed in a local or Modern Standard Arabic (MSA), and Fatwa answers that are well formed in MSA and classical Arabic (CA) [8].

- The lack of Arabic language Ontology for both MSA and CA Arabic forms [7-9].

Several FQA systems implement different QA paradigms to overcome the above challenges, including knowledge-based, IR-based and Ontology-based paradigms [7-11]. 
Knowledge-Based Question Answering (KB-QA) tackles the problem of answering NL question by first: mapping the question into some meaning representation forms such as logical rules, predicates...etc. second: uses the new form to query the structured database of Knowledge. The success of the earliest attempts in this approach is limited only to subdomains or restricted-domains. Arabic FAQ systems that adopt this approach showed considerable performance. Recent trends in this approach aim to handle broad domains by extracting the mapping knowledge automatically from the web and make use of this KB to obtain the logical query form [12].

The IR-based approach involves two methods to answer the NL questions; the first method retrieves the most similar answers from a corpus of documents that containing repeated forms of answers. This method is widely used in Fatwa QA; however, it performs well for factoid type of questions. The second method adopts summarization or abstraction techniques to extract an answer automatically from the documents corpus, this approach works well for the complex type of questions [11], however, it was not used in Fatwa QA systems, because an automatic generation of answers is not allowed in Fatwa domain due to the sensitivity of religion [2].

An Ontology-based approach is a hybrid approach that combines IR and Ontology, Ontology is a core semantic knowledge-base for many semantic-based applications. The WordNet [13] is a widely used language Ontology for English, which improve the performance of some Ontology-based QA systems to reach 94\% [14-15]. However, the Arabic language QA systems are unable to reach this level of performance because the Arabic language Ontology is not rich enough [1-9], 16-17].

Obviously, the richness properties of the Arabic language like derivational and inflectional make the construction task challenging, and that leads to the weakness of Arabic Ontology [9-17]. Another reason is that the construction task of a rich Ontology is done manually which is hard and time-consuming [18-19]. However, a semi-automatic Ontology construction approach that relies on Machine Learning (ML) techniques can minimize human effort [19-21].

Alternatively, Machine Learning (ML) approach can provide a rich semantic representation without human intervention in form of word embeddings, which is a dense vector of real values that capture the semantic representation of individual words from a large text a corpus.

However, the prior techniques in this approach either provide shallow representation at low cost as in LSA or require high computational power for rich representation as in the recurrent Neural Network [22-23]. Recent trends in ML word embeddings techniques at low computational cost able to provide a rich representation of semantic and syntactic features of words. the recent successful embeddings technique include Continuous Bag of Word (CBOW)/Skip-Gram [22] and Eigenwords [24]. These new trends have promising performance when exploited in several Natural language processing (NLP) tasks [24-27] including Arabic FQA [28].

The semantic representation for larger units like phrases, sentences and paragraphs, are also important for FQA. The composition methods combine the individual word embeddings to formulate this higher level of representations [29]. these methods can be a simple algebraic function like summation or neural networks (NN), the latter method has an encouraging performance, however, it has a complex implementation [25].

In this paper we review core and recent semantic representations techniques in KB and ML approaches that were adopted in state of the art FQA models to retrieve Arabic Fatwa answers on semantic bases.

The remainder of this paper is organized into two sections: section 2 Semantic Representations Approaches, which show the core and the recent semantic techniques for individual words in AI Knowledge-Based and Machine Learning approaches and also the Semantic Composition techniques for phrase and sentence level of representation. Section 3 Semantic-based Arabic FQA Models, which presents several models that handle 
answering Arabic Fatwa questions, based semantic techniques including Rules in Expert System, Case-based Reasoning (TCBR) in AI, Information retrieval, and Ontology.

\section{Semantic Representation Approaches}

In the Natural Language Processing (NLP), the task of representing a text meaning "Semantic" is an essential to all other computational tasks that concerning the interpretation and manipulation of text $[18,30]$. This section presents several semantic representation formulations in AI Knowledge-Based (KB) and Machine Learning (ML) approaches.

\subsection{AI Knowledge-Based (KB) Approach}

AI Knowledge representation approach aims to capture and map the essential features of objects and their relationships of the real world into a computational form of objects and relationships know as knowledge-base, which is necessary to a problem solving tasks [31]. Knowledge-base provide an efficient and highly rich semantic representation, however, the construction task is difficult and require an intensive human intervention [18] and [32-33]. Next we present several Knowledge-base representations formalisms that are widely adopted in Arabic FQA systems.

- Rule-based Formalism: In this representation formalism, a human knowledge is mapped into a logical form using hand-crafted rules like if-then rules in Expert System (ES) [31]. ES is a well known AI problem solving technique that relies on this formalism, although, it performs well in many applications including Arabic FQA the knowledge acquisition and rules construction are difficult and timeconsuming [31-34]. In Section (3.1.1) we present some of the Arabic FQA models that adopt rule-based representation formalism.

- Case Representation Formalism: a case represents knowledge about solved problem instance, and it is a "contextualized piece of knowledge representing an experience that teaches a lesson fundamental to achieving the goals of the reasoner" [35]. Cases are basically used in AI Case Based Reasoning (CBR) techniques. The construction of this representation formalism is simple and can be easily implemented in form of feature vectors or other AI representation formalisms. However, retrieving similar cases is done on keyword matching basis [33-36]. This formalism is adopted in several Arabic FQA models shown in Section (3.1.2).

- Semantic Net Formalism: Semantic Net is a graphical representation formalism, in which nodes represent semantic concepts and vertices represent the semantic relation between those concepts, the semantic similarity between concepts is assessed using path length algorithms [31]. Ontology is an important semantic KB for QA systems that handle answering questions semantically and WordNet [13] is a widely used lexical ontology for the English language. However, the manual construction of this representation is hard and expensive [30]. Section (3.3) presents some Arabic FQA models that adopt this formalism.

\subsection{Machine Learning (ML) Approach}

Instead of building an expensive semantic resource like WordNet which is not available for non-English languages, ML provides low-cost mathematical methods that follow the distributional hypothesis [37]. In this approach the semantic representation of a word is formulated in a mathematical dense vector of real values known as Word embedding, and the semantic similarity between words is specified by measuring the distance between their corresponding embeddings using geometric distance measurements like cosine distance [30]. 
This section overview several ML word embeddings techniques, including the core technique Latent Semantic Analysis (LSA), and the recent techniques: Continuous Bag of Word (CBOW)/ Skip-Gram Neural Networks (NN) and Eigenwords.

2.2.1. Latent Semantic Analysis (LSA) Technique: LSA is a mathematical statistical ML algorithm for learning the semantic representation of words and concepts that latent in a corpus of documents [38-40]. It based on Singular Value Decomposition (SVD) to extract a vector of distributional properties "word embeddings" in low dimensional space from a co-occurrence matrix of terms and documents [40]. LAS algorithm works as follows:

First: construct a reweighted co-occurrence Matrix of term-documents $X^{m, n}$, where $m$ is number of terms and $\mathrm{n}$ is number documents. Reweighting is done using the Term Frequency-Inverse Document Frequency (TF-IDF) method.

Second: Compute SVD as in equation (1) bellow to reduce the dimensionality of $\mathrm{X}$ to the most significant $k$ dimensions,

\section{$\mathrm{X} \approx \mathrm{USV}^{\mathrm{T}}$}

where $k<n$, and then the algorithm come up with three matrices $\mathrm{U}, \mathrm{S}$ and $\mathrm{V}$. the matrix $\mathrm{U}^{m, k}$ represent each term $m$ in a row vector contain $k$ concepts, $\mathrm{S}^{k, k}$ diagonal matrix, contains the $k$ most significant singular values, and the matrix $\mathrm{V}^{m, k}$ transposed, represent each document $m$ in a vector contain $k$ concepts [38].

The LSA technique has been adopted successfully in several NLP tasks [18], mainly in the Information Retrieval (IR) to perform matching between the query and the documents at a conceptual level [40], however it ignores the order of words and the context is consider only in a bag of words [24-25]. Some Arabic FQA that adopted this technique is presented in Section (3.2.1).

2.2.2 CBOW/ Skip-Gram NN Technique: In this technique, the representational embeddings of words is learned directly from large text corpus using Neural Networks (NN) [22-23]. the successful models in this approach were proposed by Mikolov, Tomas, et al., [22] they introduce two efficient word embeddings models Continuous Bag of Word (CBOW) and Skip-Gram see Figure 1. The models are scalable, fast training; unsupervised neural network models $[22,-41]$.

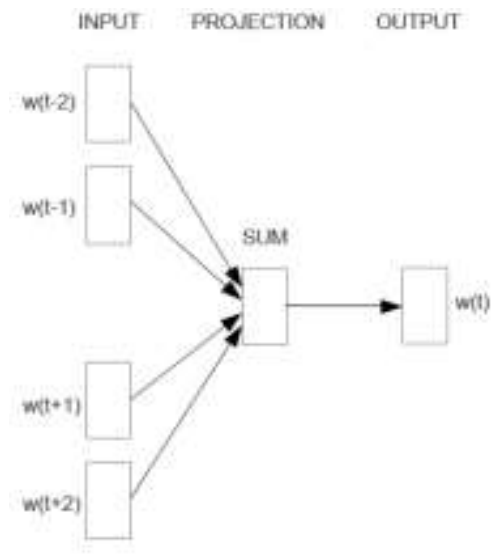

CBOW

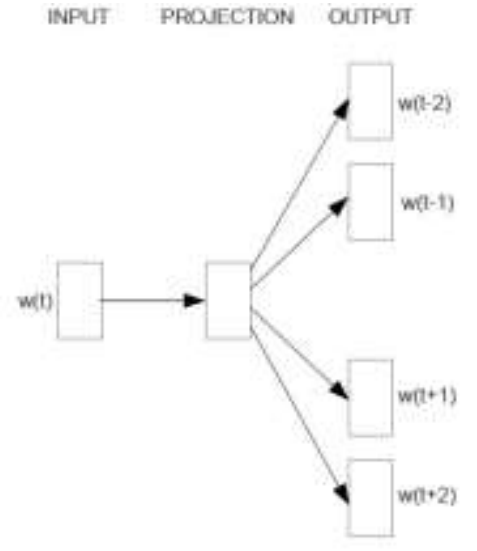

Skip-gram

Figure 1. CBOW and Skip-Gram Architectures [22]

Mikolov models were adopted successfully in many NLP tasks, for example, the Word Sense Disambiguation (WSD) [27], parsing, machine translation [25], and open domain 
QA [42], and also for Modern Standard Arabic (MSA) application such as query expansion, short answer grading and Arabic-English Vector Space Mapping [26].

For phrase level representation Mikolov models can learn the representational embeddings of the idiomatic phrases by treating words that appear more frequently together in documents and infrequently in other documents as unique tokens add to the training data [41].

Disadvantages in Mikolov models include, first: the performance depends on the parameters tuning which involve context size, number of dimensions and training algorithm [43], second: they learn a unique embedding for words of multiple senses [24]. Asingle Arabic FQA model that exploit this technique is shown in Section (3.2.1).

2.2.3 Eigenwords Technique: This technique implements spectral method to learn the implicit semantic features for words by using Eigen decompositions [44-45]. Eigenwords algorithms are proposed by Dhillon et al., [24], to estimate similar embeddings for words that have a similar context distribution, from a co-occurrence matrix of the words and their contexts. Basically, these algorithms rely on Canonical Correlation Analysis (CCA) to project the data into multi-views of low dimensional space. CCA is a dimensionality reduction technique that finds the Eigen decomposition for two sets of multi dimensional variables [24] and [44-47].

Eigenwords algorithms include: One Step Canonical Correlation Analysis (OSCCA) is efficient for the language that has enough word tokens, the Two Step Canonical Correlation Analysis (TSCCA) and ML-MVL algorithms are efficient in handling rare words problem, and Context Specific Embeddings algorithm is used to generate contextspecific embeddings, all these algorithms initially work similarly ,as the following:

First: partition the whole text corpus into fixed size segments see Figure 2, each segment contains single word and its context with a length of $\mathrm{h}$ words to the left and $\mathrm{h}$ words right.

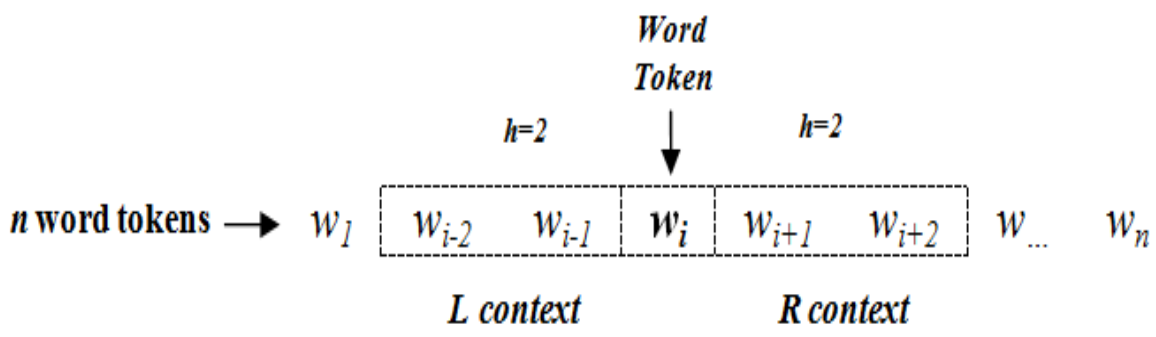

Figure 2. Defines the Left and Right Contexts of Token $w_{i}$ with Context Size of $2 \mathrm{~h}$ Words Tokens.

Second: using the one-hot encoding representation the algorithms construct a word matrix $\mathrm{W}^{n, v}$ containing $n$ row vectors for the vocabulary words with length $v$ and also construct a context matrix $\mathrm{C}^{n, 2 h v}$ containing $n$ row vectors with length $2 h v$ for the LR contexts corresponding to each word in $\mathrm{W}$.

Third: compute the Eigen decomposition using the CCA as in Equation (2) bellow,

$\mathrm{U}, \mathrm{V}=\mathrm{CCA}\left(\mathrm{W}^{\prime}, \mathrm{C}^{\prime}\right)$

where $\mathrm{W}^{\prime}$ and $\mathrm{C}^{\prime}$ are co-occurrence matrices for the words and their contexts. $\mathrm{U}^{n, w}$ and $\mathrm{V}^{n, c}$ matrices contain the embeddings for the $n$ words and $\mathrm{n}$ contexts reduced to $w$ and $c$ dimensions respectively.

Eigenwords techniques based on a strong theoretical foundation provided several efficient solutions for the semantic representation of individual words including the rare words problem, in a fast and scalable manner [24-47]. 


\subsection{Semantic Composition}

Individual words representation approaches mentioned in section (2.1 and 2.2) are not suitable for sentence level representation, because there no sentence distribution [29]. The sentence level representation is formed from the embeddings for individual words of the sentence using a composition function; simple algebraic functions and Neural Networks are the most commonly used in this task [18]. Algebraic function like addition or multiplication [48] are simple and fast, but fails to capture important aspects such as grammatical relations, words order, and ambiguity [29].

In Neural Networks composition functions [30] there is several research works adopt different NN techniques including Recursive Neural Networks and Convolutional Neural Networks [29]. In most cases these models able to preserves the syntactic and semantic features of a sentence, however their computational cots is very high [25].

\section{Semantic-based Arabic FQA Models}

In this section, we review state of the art FQA models that exploit different semantic techniques to overcome the challenges of answering Arabic Fatwa questions, using various QA paradigms including knowledge-Based (KB), Information Retrieval (IR) and Ontology-based. Table 1 presents a comparative summary of the QA paradigms, semantic techniques, performance, Fatwa scope and limitations of various FQA models showed next.

\subsection{Knowledge-based FQA Models}

Knowledge-based QA models provide answers for NL question based on well-defined forms of the human Knowledge representation. Rule-based Expert System and Casebased Reasoning (CBR) are techniques that attract several researchers to adopt techniques in handling Arabic FQA problem. The next two sub sections present some of these models.

3.1.1. Rule-based Expert System (RBES): Rule-based expert system (RBES) is one of the oldest knowledge-based techniques that have a good reputation when applied in several sub-domain problems including medical diagnosis, QA and FQA [31-49].

As an example; Suliman et al., [49] adopt the RBES in FQA to answer hajj pilgrim's questions during hajj rituals. Rules are being used to represent the knowledge and the answers are concluded by inference engine after examining Knowledge-base using forward chaining. Although the model is a web-based prototype, it did not support natural language query interface [49].

Another RBES model for FQA is developed by Afaf et al., [50] for answering questions about Zakat; which is a specific amount of money taken from rich Muslims to be given to their poor ones; it calculates the different types of Zakat and specifies the amount required to be paid. The model captures Zakat knowledge from human experts. However, the user interface is not friendly, and did not support Arabic language [50].

Although RBES is stable and reduce cost and risk, and its good performance in Fatwa QA, there is no easy way to be applied in multiple Fatwa domains due to the challenges of knowledge acquisition and interpretation [49].

3.1.2. Case-Based Reasoning (CBR): CBR is a promising AI Knowledge-Based technique. CBR is "reasoning by remembering, where previously solved cases are used to suggest solutions for novel but similar problems" [34]. It is increasingly used in advising and QA [51]. The general CBR working cycle [52] consist of four steps, shown in Figure 3.

1) RETRIEVE the most similar case(s); 
2) REUSE the case(s) to attempt to solve the current problem;

3) REVISE the proposed solution if necessary;

4) RETAIN the new solution as a part of a new case.

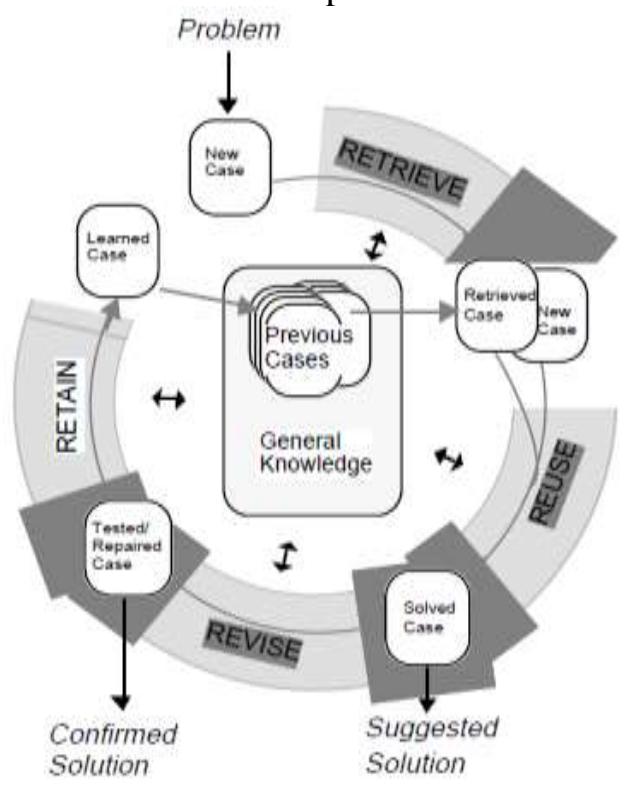

Figure 3. CBR Working Cycle [52]

Recently CBR is adopted in several Arabic FQA systems; for example, Nouaouria et al., [53] developed a CBR search tool oriented to help Mufti in preparing fast an accurate Fatwa answer for a new Fatwa Question, by reusing the Fatwa of past situations of a similar problem. The tool can be used by scholars and experts domain. However it is restricted to drinking and smoking fatwa category [53].

Elhalwany et al., [2] based on CBR developed FQA system for Egyption Dar al-Ifta. The system allows the user to use a similar cases search tool to look for similar questions or to submit the question to Dar al-Ifta domain experts. The system automatically discovers and utilizes textual cases and also the similarity knowledge. However, it ignores word order and negation [2].

Although CBR has many advantages as mentioned in Section (2.1), it also has some disadvantage including the lack of semantic mechanism and the absence of relations between cases [2], these limitation prevents several of CBR models to exceed accuracy of $53 \%$ [33]. However, the performance of CBR can be enhanced by integrating ontology with traditional CBR [34], [51-54].

\subsection{IR-based FQA Models}

The introducing of IR technique to the QA problem in Text REtrieval Conference (TREC) 1999, allows research studies to tackle open domain QA [9], QA systems that rely on IR model achieved significant success in QA systems that support English language $[10,55]$. The basic components of IR-based QA as shown in Figure 4 include, first Question analysis, to specify the question type and answer type is detected and the query is reformulated before it send to the search engine, second Document/passage retrieval to retrieve and rank passages, and third answer extraction to extract candidate answers [11]. 


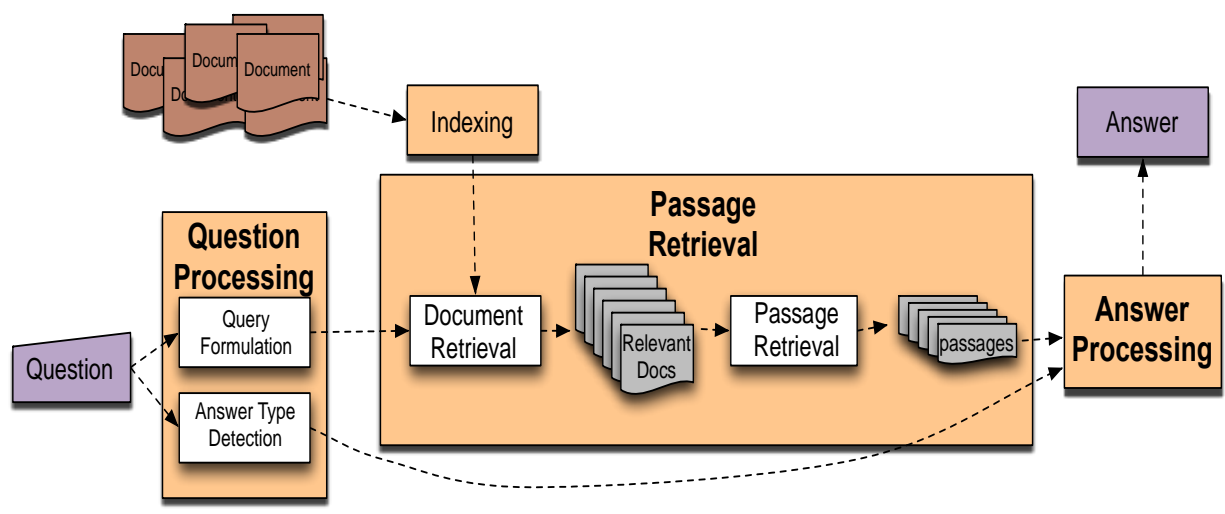

Figure 4. IR Based QA Architecture [11]

In IR-based models, the word embedding is the basic semantic representation form. On this basis, the IR-based Arabic FQA models are grouped into two classes the LSA and CBOW/ SKIP-G NN embeddings as follows:

3.2.1. LSA Embeddings: In this category Arabic FQA web sites with IR capability retrieve the answer from a set documents [4-6] and [56-57]. The main disadvantage in these models is that the answers retrieval can involves irrelevant answers; however ranking the most relevant above the irrelevant answers is a challenging task in this category [8].

Recent trend in this category is a Community Question Answering (CQA) websites like Yahoo! Answers, Google, Stackoverflow [58] is increasingly popular which combines IR model and social network techniques. CQA allows the user to pose a question in natural language and expert users pose some good answers [59]. In this technique the ranking or selecting the most relevant answers is a challenging task. Some attempts try to address the CQA answer selection problem in Arabic Fatwa however the performance of the best attempts [60] unable to reach the accuracy of 50\% in SemEval2015 Task 3 [8] and [59].

1.1.1 CBOW/ SKIP-G NN Embeddings: QA models in this category are based on an efficient word embeddings constructed by SKIP-G and CBOW Neural Networks [22], and because it is recently proposed, there is a small number of research studies have investigated in these techniques particularly for Arabic FQA [42].

One of the seldom researches that exploit this technique in Arabic FQA is Vector SLU model introduced by Belinkov at el., [28]. The model induces words embedded using Mikolov model [22], trained on the Arabic Gig word corpus, the model is the second winner with accuracy $76.32 \%$ in the task of Answer Selection in CQA-Arabic Fatwa domain for SemEval-2015 Task 3 [59].

\subsection{Hybrid FQA Models}

In a hybrid QA approach that combines Knowledge-Base (KB) and Information Retrieval (IR) techniques, as an example for hybrid QA models see Figure 5, Ontology is the most important Knowledge resource of semantics [15], which is "a formal, explicit specification of a shared conceptualization" [61], it provides the semantics of terms and concepts and semantic relation between them in form of electronic resource that can be shared by human and machines, which allows machine to understand semantics of textual human NL [62]. 


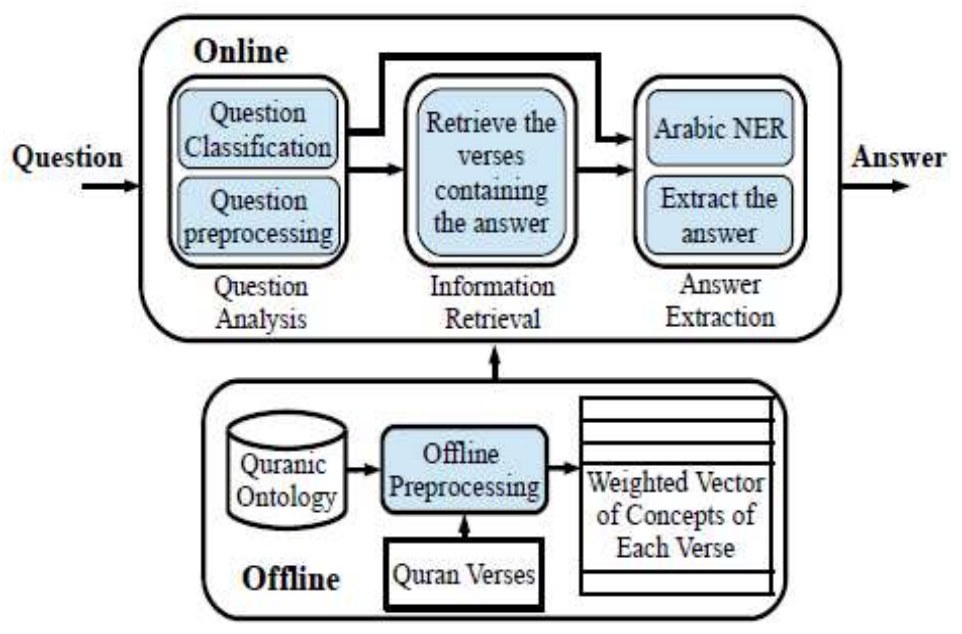

Figure 5. Al-Bayan Model Architecture [7]

An optimal performance is obtained in Ontology-based FQA system by adopting language Ontology that is manually constructed by human experts such as WordNet [13], a widely popular lexical ontology for English; however the construction task of ontology is tedious and expensive [19]. To overcome the hardness construction of a language ontology, several attempts try to build non-English language Ontology based on the English WordNet, however these attempts could not reach their goals because languages have different set of lexicalized concepts [63], Arabic WordNet [64] is an example of an ontology that has been built upon WordNet [65].

Ontology learning is an alternative low-cost technique for building ontology in automated or semi-automated ways by applying Linguistic and ML methods on a large text corpus [20-21]. This approach is commonly used in constructing domain ontology that oriented for specific domain like Medical or Fatwa. Although this approach could not build a rich ontology [19] the resulted ontology has a significant improvement in the performance of application focus on Arabic Fatwa like in [66].

Ontology-based Models showed a better performance in understanding the user queries and hence the retrieval performance [67]. However, as a result to the lack of a rich Arabic language Ontology [7-16] and [68], most researches studies in Ontology-Based FQA focus on specific domain Ontologys that have been built manually or automatically. Next we preset some of these studies.

Heba at el., [7] proposed "Al-Bayan Arabic Question Answering System for the Holy Quran" see Figure 5. It was the first Fatwa Question Answering system that adopts manually constructed Quran Ontology and IR techniques to answer user questions from the Quran and its interpretation books. The system achieves 85\% accuracy for only type of question "Quran interpretation".

Al-Bayan also introduced another model that uses Quran Ontology built in [7] to construct vector representation of ontology concepts for each question and its candidate answers in Fatwa corpus, and it find a semantic similarity scores in addition to information retrieval scores for each candidate answer, and then fed both scores into a decision tree to classify each candidate answer [69]. The model achieved $74.53 \%$ accuracy when tested in the task of Answer Selection in CQA-Arabic Fatwa domain in SemEval-2015 Task 3 [59].

Due to the difficulties involved in manually constructing Arabic Ontology, [1-16] and [68] some research initiatives adopt automatic techniques to build domain ontology. For example MUSTEFA al. et., [66] proposed a hybrid QA model (ontology +IR) for FQA see Figure 6, the domain ontology was constructed in semi-automated way using Term Frequency-Inverse Document Frequency (TFIDF) approach, the QA corpus involves 
1049 question-answer pair in Salah category answered by Ibn Uthaymeen expert domain. The model achieves the performance of $90 \%$ using F-measure. However test dataset consist of only 30 new questions [66].

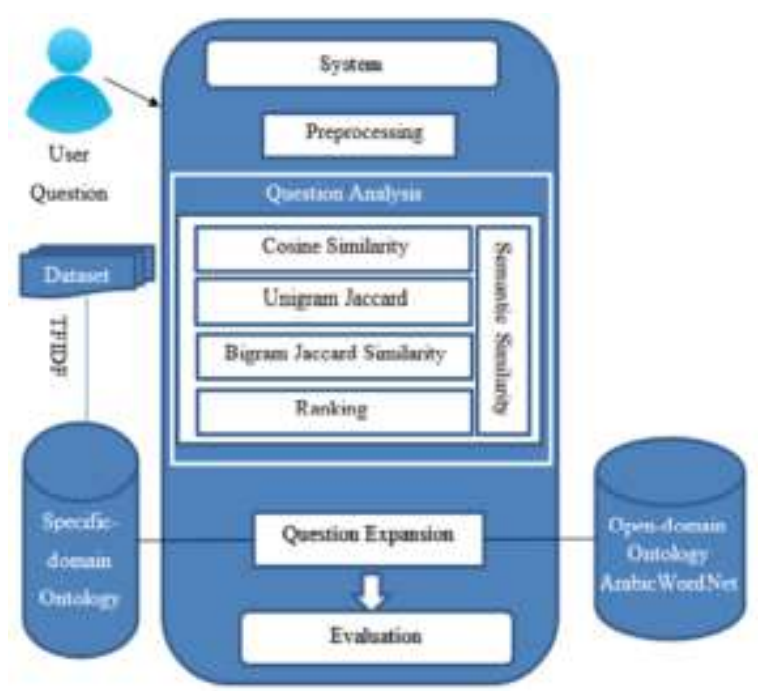

Figure 6. Salah Ontology-based FQA Model Architecture [66]

The summary of this review See Table 1, shows that the KB FQA models could not easily scale to multiple Fatwa categories and the recent KB-QA paradigm that relies on the open web KB did not attract researchers in the field of FQA area.

In IR-based QA paradigm, the recent form "CQA" in FQA inherited the limitations of retrieving irrelevant answers; however, adding Ontology improved CQA performance. On the other side of IR-Based QA paradigm, the recent CBOW/SKIP-G embedding techniques have comparable performance to an Ontology technique in multiple Fatwa categories for the task of answer selection.

\section{Table 1. Comparison between Semantic-Based Arabic FQA Approaches and Techniques}

\begin{tabular}{|c|c|c|c|c|c|c|}
\hline $\begin{array}{c}\text { QA } \\
\text { Paradigms }\end{array}$ & $\begin{array}{l}\text { Semantic } \\
\text { Techniques }\end{array}$ & $\begin{array}{l}\text { FQA } \\
\text { Models }\end{array}$ & Description & $\begin{array}{c}\text { Fatwa } \\
\text { Category } \\
\text { (single/ } \\
\text { Multiple) }\end{array}$ & Accuracy & Limitations \\
\hline \multirow[b]{2}{*}{$\begin{array}{c}\text { Knowledge- } \\
\text { Based }\end{array}$} & \multirow[b]{2}{*}{$\begin{array}{c}\text { Rule-Based } \\
\text { (RBES) }\end{array}$} & $\begin{array}{l}\text { Sulaiman } \\
\text { et al., [49] }\end{array}$ & $\begin{array}{l}\text { Answering } \\
\text { pilgrim } \\
\text { questions } \\
\text { during the hajj } \\
\text { rituals }\end{array}$ & $\begin{array}{l}\text { Single } \\
\text { (Hajj) }\end{array}$ & NA & $\begin{array}{l}\text { - Evaluation } \\
\text { was not } \\
\text { done. } \\
\text { - Arabic NL } \\
\text { query not } \\
\text { supported. }\end{array}$ \\
\hline & & $\begin{array}{l}\text { ZAKAT } \\
\text { ES } \\
\text { Al-Riyami } \\
\text { et al., [50] }\end{array}$ & $\begin{array}{l}\text { Provides } \\
\text { answers for } \\
\text { different types } \\
\text { of Zakat } \\
\text { questions by } \\
\text { calculating the } \\
\text { required } \\
\text { amount to be } \\
\text { paid. }\end{array}$ & $\begin{array}{l}\text { Single } \\
\text { (Zakat) }\end{array}$ & $90 \%$ & $\begin{array}{l}\text { The Arabic } \\
\text { NL query } \\
\text { not } \\
\text { supported. }\end{array}$ \\
\hline
\end{tabular}




\begin{tabular}{|c|c|c|c|c|c|c|}
\hline & \multirow[t]{2}{*}{$\begin{array}{l}\text { Cased-Based } \\
\text { (CBR) }\end{array}$} & $\begin{array}{l}\text { Nouaouria } \\
\text { et al., [53] }\end{array}$ & $\begin{array}{l}\text { A Utility tool } \\
\text { for the Mufti } \\
\text { to help him in } \\
\text { answering a } \\
\text { new Fatwa } \\
\text { question } \\
\text { correctly } \\
\text { based on } \\
\text { previous } \\
\text { similar cases. }\end{array}$ & $\begin{array}{c}\text { Single } \\
\text { (Drinking) }\end{array}$ & NA & $\begin{array}{l}\text { - Used by } \\
\text { scholars and } \\
\text { experts only } \\
\text { - Not } \\
\text { supported } \\
\text { Arabic NL } \\
\text { query. }\end{array}$ \\
\hline & & $\begin{array}{l}\text { Elhalwany } \\
\text { et al., [2] }\end{array}$ & $\begin{array}{l}\text { A similar } \\
\text { case-based } \\
\text { search tool for } \\
\text { retrieving } \\
\text { similar Fatwa } \\
\text { answers. }\end{array}$ & $\begin{array}{l}\text { Single } \\
\text { (the } \\
\text { worship } \\
\text { Fatwa) }\end{array}$ & NA & $\begin{array}{l}\text {-no } \\
\text { semantics } \\
\text { mechanism } \\
\text { used. } \\
\text {-Efficiency } \\
\text { decreases } \\
\text { when data } \\
\text { get large. }\end{array}$ \\
\hline \multirow[b]{2}{*}{ IR-Based } & $\begin{array}{c}\text { LSA } \\
\text { Embeddings }\end{array}$ & {$[4-6,56-57]$} & $\begin{array}{l}\text { A web site } \\
\text { with search } \\
\text { tool about } \\
\text { pervious } \\
\text { answered } \\
\text { Fatwa. }\end{array}$ & Multiple & NA & $\begin{array}{l}\text {-ignores order } \\
\text { of words. } \\
\text {-retrieves } \\
\text { relevant and } \\
\text { irrelevant } \\
\text { answers. }\end{array}$ \\
\hline & $\begin{array}{c}\text { NN } \\
\text { Embeddings } \\
\text { CBOW/SKIP- } \\
\text { G }\end{array}$ & $\begin{array}{l}\text { Belinkov et } \\
\text { al., [28] }\end{array}$ & $\begin{array}{l}\text { A semantic- } \\
\text { based QA } \\
\text { system, for } \\
\text { task of } \\
\text { Answer } \\
\text { Selection in } \\
\text { CQA-Arabic } \\
\text { Fatwa domain } \\
\text { in SemEval- } \\
\text { 2015 Task } 3 \\
\text { [39]. }\end{array}$ & Multiple & $76.32 \%$ & $\begin{array}{l}\text { For each } \\
\text { new } \\
\text { question, it } \\
\text { is only } \\
\text { classify } 5 \\
\text { answers as: } \\
\text { Direct, } \\
\text { Related or } \\
\text { Irrelevant. }\end{array}$ \\
\hline \multirow{2}{*}{$\begin{array}{l}\text { Ontology- } \\
\text { Based }\end{array}$} & \multirow{2}{*}{$\begin{array}{l}\text { Semantic-net } \\
\text { Manually } \\
\text { built } \\
\text { Ontology }\end{array}$} & $\begin{array}{l}\text { Al-Bayan } \\
\text { Abdelnasser } \\
\text { et al., [7] }\end{array}$ & $\begin{array}{l}\text { Semantic- } \\
\text { based QA for } \\
\text { answering } \\
\text { question about } \\
\text { Quran by } \\
\text { retrieving the } \\
\text { verses } \\
\text { interpretation. }\end{array}$ & $\begin{array}{c}\text { Single } \\
\text { (Quran } \\
\text { Taffseer) }\end{array}$ & $85 \%$ & $\begin{array}{l}\text {-only answer } \\
\text { factoid } \\
\text { questions } \\
-59 \text { questions } \\
\text { to test and } \\
\text { evaluate the } \\
\text { model. }\end{array}$ \\
\hline & & $\begin{array}{l}\text { Al-Bayan } \\
\text { Mohamed } \\
\text { et al., [69] }\end{array}$ & $\begin{array}{l}\text { A semantic- } \\
\text { based QA for } \\
\text { task of } \\
\text { Answer } \\
\text { Selection in } \\
\text { CQA-Arabic } \\
\text { Fatwa domain } \\
\text { in SemEval- } \\
\text { 2015 Task } 3\end{array}$ & Multiple & $74.53 \%$ & $\begin{array}{l}\text { For each } \\
\text { new } \\
\text { question, it } \\
\text { is only } \\
\text { classify } 5 \\
\text { answers as: } \\
\text { Direct, } \\
\text { Related or } \\
\text { Irrelevant. }\end{array}$ \\
\hline
\end{tabular}




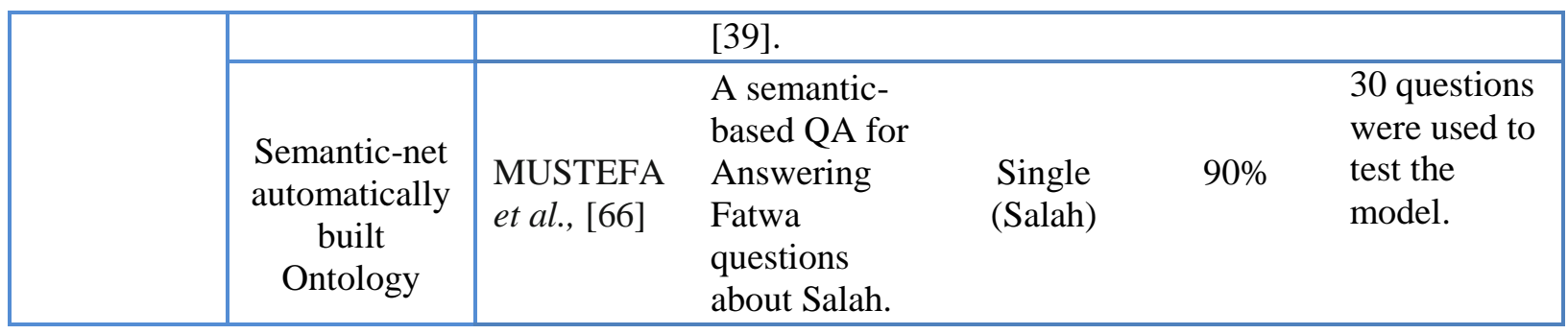

\section{Conclusion}

This paper presented a review that covered several semantic-based Arabic FQA models in the Islamic religion domain "Fatwa", and their underlying semantics techniques including Rules in ES, Cases in CBR, semantic net in Ontology and LSA, CBOW/SKIPG, Eigenwords embeddings in IR. Moreover, the review highlighted the new directions and the open issues in Arabic FQA paradigms including KB, IR-based, and ontologybased.

Although the construction of Arabic ontology is a crucial challenge, it remains the core semantic resource for the IR-based FQA models. These models are increasingly becoming more popular. Furthermore, to obtain a better performance, a domain ontology for Hadith is needed besides the Quran ontology. On the other side, the adoption of the recent semantic techniques such as "Skip-Gram/CBOW" NN embeddings in some tasks of Arabic FQA revealed a promising performance compared to the core semantic technique "Arabic Ontology". These open issues encouraged researchers to investigate the adoption of the recent semantic techniques in handling other tasks of Arabic FQA for future works.

\section{References}

[1] M. AlMaayah, M. Sawalha and M. Abushariah, "A proposed model for Quranic Arabic WordNet", in Proceedings of the 2nd Workshop on Language Resources and Evaluation for Religious Texts, 31 May 2014, Reykjavik, Iceland, (2014), pp. 9-13.

[2] I. Elhalwany, A. Mohammed, K. Wassif and H. Hefny, "Using Textual Case-based Reasoning in Intelligent Fatawa QA System", The International Arab Journal of Information Technology, vol. 12, (2015).

[3] fatwa.islamweb.net/fatwa/index.php. (2016) May.

[4] www.islam-qa.com. (2016) May.

[5] www.alifta.com. (2016) May.

[6] www.dar-alifta.org. (2016) May.

[7] H. Abdelnasser, R. Mohamed, M. Ragab, A. Mohamed, B. Farouk, N. El-Makky and M. Torki, "AlBayan: an arabic question answering system for the holy quran", ANLP 2014, (2014), pp. 57.

[8] P. Nakov, L. Màrquez, A. Moschitti, W. Magdy, H. Mubarak, A. Freihat, J. Glass and B. Randeree, "SemEval-2016 task 3: Community question answering", in Proceedings of the 10th International Workshop on Semantic Evaluation. San Diego, California. Association for Computational Linguistics, (2016).

[9] M. Shaheen and A. M. Ezzeldin, "Arabic question answering: Systems, resources, tools, and future trends", Arabian Journal for Science and Engineering, vol. 39, (2014), pp. 4541-4564.

[10] P. Molino, P. Basile, A. Caputo, P. Lops and G. Semeraro, "Exploiting distributional semantic models in question answering", in Semantic Computing (ICSC), 2012 IEEE Sixth International Conference on, (2012), pp. 146-153.

[11] D. Jurafsky and J. H. Martin, Speech and Language Processing: An Introduction to Natural Language Processing, Computational Linguistics, and Speech Recognition: Prentice Hall, (2009).

[12] J. Bao, N. Duan, M. Zhou and T. Zhao, "Knowledge-based question answering as machine translation", (2014).

[13] G. A. Miller, "WordNet: a lexical database for English", Communications of the ACM, vol. 38, (1995), pp. 39-41.

[14] P. Raj, "Architecture of an Ontology-Based Domain-Specific Natural Language Question Answering System", arXiv preprint arXiv:1311.3175, (2013).

[15] I. Zitouni, "Natural language processing of semitic languages", Springer, (2014).

[16] H. U. Khan, S. M. Saqlain, M. Shoaib and M. Sher, "Ontology based semantic search in Holy Quran", International Journal of Future Computer and Communication, vol. 2, (2013), pp. 570. 
[17] W. Black, S. Elkateb, H. Rodriguez, M. Alkhalifa, P. Vossen, A. Pease and C. Fellbaum, "Introducing the Arabic wordnet project", in Proceedings of the third international WordNet conference, (2006), pp. 295-300.

[18] J. Mitchell and M. Lapata, "Composition in distributional models of semantics", Cognitive Science, vol. 34, (2010), pp. 1388-1429.

[19] A. A. Barforush and A. Rahnama, "Ontology learning: revisted", Journal of Web Engineering, vol. 11, (2012), pp. 269-289.

[20] A. Al-Arfaj and A. Al-Salman, "Ontology Construction from Text: Challenges and Trends".

[21] M. Hazman, S. R. El-Beltagy and A. Rafea, "A survey of ontology learning approaches", database, vol. 7, (2011), pp. 6.

[22] T. Mikolov, K. Chen, G. Corrado and J. Dean, "Efficient estimation of word representations in vector space", arXiv preprint arXiv:1301.3781, (2013).

[23] M. Baroni, G. Dinu and G. Kruszewski, "Don't count, predict! A systematic comparison of contextcounting vs. context-predicting semantic vectors", in ACL no. 1, (2014), pp. 238-247.

[24] P. S. Dhillon, D. P. Foster and L. H. Ungar, "Eigenwords: Spectral word embeddings", The Journal of Machine Learning Research, vol. 16, (2015), pp. 3035-3078.

[25] Q. V. Le and T. Mikolov, "Distributed Representations of Sentences and Documents", in ICML, (2014), pp. 1188-1196.

[26] M. A. Zahran, A. Magooda, A. Y. Mahgoub, H. Raafat, M. Rashwan and A. Atyia, "Word Representations in Vector Space and their Applications for Arabic", in International Conference on Intelligent Text Processing and Computational Linguistics, (2015), pp. 430-443.

[27] X. Chen, Z. Liu and M. Sun, "A Unified Model for Word Sense Representation and Disambiguation", in EMNLP, (2014), pp. 1025-1035.

[28] Y. Belinkov, M. Mohtarami, S. Cyphers and J. Glass, "VectorSLU: A continuous word vector approach to answer selection in community question answering systems", SemEval-2015, (2015), pp. 282.

[29] P. Blunsom, E. Grefenstette and K. M. Hermann, "New directions in vector space models of meaning", in Proceedings of the 52nd Annual Meeting of the Association for Computational Linguistics, (2014)

[30] K. M. Hermann, "Distributed Representations for Compositional Semantics", arXiv preprint arXiv:1411.3146, (2014).

[31] E. Rich and K. Knight, "Artificial intelligence", McGraw-Hill, New, (1991).

[32] P. Lucas and L. V. Der Gaag, "Principles of expert systems", Citeseer, (1991).

[33] S. H. El-Sappagh and M. Elmogy, "Case Based Reasoning: Case Representation Methodologies", International Journal of Advanced Computer Science \& Applications, vol. 1, (2014), pp. 192-208.

[34] R. C. Schank, "Dynamic memory: A theory of reminding and learning in computers and people", Cambridge University Press, (1983).

[35] J. Kolodner and C.-B. Reasoning, "Morgan Kauffman Publishers", Inc., San Mateo, CA, (1993).

[36] I. Watson and F. Marir, "Case-based reasoning: A review", Knowledge Engineering Review, vol. 9, (1994), pp. 327-354.

[37] Z. S. Harris, "Distributional structure", Word, vol. 10, (1954), pp. 146-162.

[38] T. K. Landauer, P. W. Foltz and D. Laham, "An introduction to latent semantic analysis", Discourse processes, vol. 25, (1998), pp. 259-284.

[39] P. D. Turney and P. Pantel, "From frequency to meaning: Vector space models of semantics", Journal of artificial intelligence research, vol. 37, (2010), pp. 141-188.

[40] S. Deerwester, S. T. Dumais, G. W. Furnas, T. K. Landauer and R. Harshman, "Indexing by latent semantic analysis", Journal of the American society for information science, vol. 41, (1990), pp. 391.

[41] T. Mikolov and J. Dean, "Distributed representations of words and phrases and their compositionality", Advances in neural information processing systems, (2013).

[42] P. Molino and L. M. Aiello, "Distributed Representations for Semantic Matching in non-factoid Question Answering", in SMIR@ SIGIR, (2014), pp. 38-45.

[43] T. Mikolov and O. H. Way, "Using Neural Networks for Modeling and Representing Natural Languages", in COLING (Tutorials), (2014), pp. 3-4.

[44] K. Kamvar, S. Sepandar, K. Klein, D. Dan, M. Manning and C. Christopher, "Spectral learning", in International Joint Conference of Artificial Intelligence, (2003).

[45] S. B. Cohen, K. Stratos, M. Collins, D. P. Foster and L. H. Ungar, "Spectral Learning of Latent-Variable PCFGs", in ACL (1), (2012), pp. 223-231.

[46] M. Borga, "Canonical correlation: a tutorial", On line tutorial http://people. imt. liu. se/magnus/cca, vol. 4, (2001), pp. 5.

[47] D. Osborne, S. Narayan and S. B. Cohen, "Encoding Prior Knowledge with Eigenword Embeddings", arXiv preprint arXiv:1509.01007, (2015).

[48] J. Mitchell and M. Lapata, "Vector-based Models of Semantic Composition", in ACL, (2008), pp. 236244.

[49] S. Sulaiman, H. Mohamed, M. R. M. Arshad, A. Ahmad and S. Sulaiman, "A knowledge-based approach to facilitate queries by hajj pilgrims", in 2008 International Symposium on Information Technology, (2008), pp. 1-9. 
[50] A. Al-Riyami, A. A.-H. K. Al-Amri and K. A. Al-Busaidi, "Zakat Expert System", vol. 1, (2014), pp. 31.

[51] P. Han, R.-M. Shen, F. Yang and Q. Yang, "The application of case based reasoning on Q\&A system", in Australian Joint Conference on Artificial Intelligence, (2002), pp. 704-713.

[52] A. Aamodt and E. Plaza, "Case-based reasoning: Foundational issues, methodological variations, and system approaches", AI communications, vol. 7, (1994), pp. 39-59.

[53] N. Nouaouria, F. Atil, M. Laskri, D. Bouyaya and A. H. Amari, "A Cased Based Tool as Intelligent Assistance to Mufti", Arabian Journal for Science and Engineering, vol. 31, (2006), pp. 75-90.

[54] V. Ceausu and S. Desprès, "A semantic case-based reasoning framework for text categorization", in The Semantic Web, ed: Springer, (2007), pp. 736-749.

[55] D. Ferrucci, E. Brown, J. Chu-Carroll, J. Fan, D. Gondek, A. A. Kalyanpur, A. Lally, J. W. Murdock, E. Nyberg and J. Prager, "Building Watson: An overview of the DeepQA project", AI magazine, vol. 31, (2010), pp. 59-79.

[56] www.alifta.com. (2015) May..

[57] fatwa.islamweb.net. (2015) May.

[58] Z. Zolaktaf, F. Riahi, M. Shafiei and E. Milios, "Modeling Community Question-Answering Archives", ed.

[59] P. L. W. AlessandroMoschitti, J. Glass and B. Randeree, "Semeval-2015 task 3: Answer selection in community question answering", SemEval-2015, (2015), pp. 269.

[60] M. Nicosia, S. Filice, A. Barrón-Cedeno, I. Saleh, H. Mubarak, W. Gao, P. Nakov, G. Da San Martino, A. Moschitti and K. Darwish, "QCRI: Answer selection for community question answeringexperiments for Arabic and English", in Proceedings of the 9th International Workshop on Semantic Evaluation, SemEval, (2015), pp. 203-209.

[61] T. R. Gruber, "Toward principles for the design of ontologies used for knowledge sharing?", International journal of human-computer studies, vol. 43, (1995), pp. 907-928.

[62] Y. Rezgui, "Text-based domain ontology building using tf-idf and metric clusters techniques", The Knowledge Engineering Review, vol. 22, (2007), pp. 379-403.

[63] F. Bond, C. Fellbaum, S. K. Hsieh, C.-R. Huang, A. Pease and P. Vossen, "A multilingual lexicosemantic database and ontology", in Towards the Multilingual Semantic Web, ed: Springer, (2014), pp. 243-258.

[64] S. Elkateb, W. Black, H. Rodríguez, M. Alkhalifa, P. Vossen, A. Pease and C. Fellbaum, "Building a wordnet for arabic", in Proceedings of The fifth international conference on Language Resources and Evaluation (LREC 2006), (2006).

[65] E. Kamal, M. Rashwan and S. Alansary, "High Quality Arabic Lexical Ontology Based on MUHIT, WordNet, SUMO and DBpedia", in International Conference on Intelligent Text Processing and Computational Linguistics, (2015), pp. 98-111.

[66] M. Sheker, S. Saad, R. Abood and M. Shakir, "Domain-specific ontology-based approach for Arabic question answering", Journal of Theoretical and Applied Information Technology, vol. 83, (2016), pp. 43.

[67] L. Al-Safadi, D. Al-Rgebh and W. AlOhali, "A comparison between ontology-based and translationbased semantic search engines for Arabic blogs", Arabian Journal for Science and Engineering, vol. 38, (2013), pp. 2985-2992.

[68] N. H. Abbas, "Quran'search for a concept'tool and website", Unpublished thesis, University of Leeds, (2009).

[69] R. Mohamed, M. Ragab, H. Abdelnasser, N. M. El-Makky and M. Torki, "Al-Bayan: A knowledgebased system for Arabic answer selection", SemEval-2015, (2015), pp. 226. 